www.nature.com/ejhg

\title{
A single mutated $M E F V$ allele in Israeli patients suffering from familial Mediterranean fever and Behçet's disease (FMF-BD)
}

\author{
Avi Livneh ${ }^{* 1}$, Ivona Aksentijevich ${ }^{2}$, Pnina Langevitz ${ }^{1}$, Yelizaveta Torosyan ${ }^{2}$, \\ Nitza G-Shoham ${ }^{1}$, Yael Shinar ${ }^{1}$, Elon Pras ${ }^{1}$, Nurit Zaks ${ }^{1}$, Shai Padeh $^{1}$, Daniel L Kastner ${ }^{2}$ and \\ Mordechai $\operatorname{Pras}^{1}$
}

\author{
${ }^{1}$ Heller Institute of Medical Research, Sheba Medical Center, Tel-Hashomer, and Sackler Faculty of Medicine Tel \\ Aviv University, Tel Aviv, Israel; ${ }^{2}$ Genetics Section, Arthritis and Rheumatism Branch, National Institute of \\ Arthritis and Musculoskeletal and Skin Diseases, Bethesda, Maryland, USA
}

\begin{abstract}
Although familial Mediterranean fever (FMF) is an autosomal recessive disorder, preliminary partial mutation analysis suggested that about $60 \%$ of FMF patients, who also suffer from Behçet's disease (FMF-BD), have only a single mutated FMF gene (MEFV). In this study, the possibility that patients with FMF-BD may indeed be carriers of a single mutated MEFV is further analysed. The presence of mutations in the coding region of $M E F V$ of eight patients with FMF-BD, representing six families with 47 members, was determined by sequencing. A possible role for the non-carrier chromosome and for BD in the expression of FMF in patients with a single mutated MEFV allele was determined by analysing the association between these variables and the presence of FMF in heterozygous kin. Sequence analysis revealed that all eight patients had indeed only one mutation in the coding region of MEFV. The patients' non-carrier chromosomes converged into three different MEFV haplotypes and were shared by heterozygous unaffected kin in five of six families. BD was found in 10 of 11 carriers with FMF vs one of 16 carriers without FMF $(P<0.001)$. These results suggest that FMF may be expressed in individuals harbouring only one coding mutation in MEFV. The findings argue against a role for the non-carrier chromosome in the induction of FMF, and suggest that the FMF phenotype in this cohort was associated with the simultaneous presence of BD. These findings may mirror a more generalised rule, that FMF may be precipitated in carriers of a single mutated FMF gene by factors unrelated to the other MEFV allele. European Journal of Human Genetics (2001) 9, $191-196$.
\end{abstract}

Keywords: familial Mediterranean fever; Behçet's disease; MEFV; mutations; single nucleotide polymorphism; chromosome

\section{Introduction}

Familial Mediterranean fever (FMF) is an ethnically restricted, autosomal recessive disease, usually caused by missense mutations in $M E F V$, the gene encoding pyrin/ marenostrin. ${ }^{1,2}$ The disease is characterised by short, irregular and febrile bouts of painful manifestations in one or more

${ }^{*}$ Correspondence: Professor Avi Livneh, MD, Heller Institute, Sheba Medical Center, Tel-Hashomer 52621, Israel. Tel: +972 3 5302454, Fax: +972 3 5307002, E-mail: alivneh@post.tau.ac.il

Received 9 August 2000; revised 3 November 2000; accepted 15 November 2000 body sites, presenting usually as peritonitis, arthritis, pleuritis and erysipeloid erythema and a propensity to develop amyloidosis. ${ }^{3-5}$ Some vasculitides, including Henoch-Schönlein purpura and polyarteritis nodosa, are more commonly found in FMF. ${ }^{4}$

Behçet's disease (BD) is an inflammatory genetic disease of episodic nature, in which vasculitis plays an important pathogenic role. Although tightly linked to HLA-B5, ${ }^{67}$ neither the genetic defect nor the mode of inheritance are currently known. The clinical picture of BD is broad and may include recurrent aphthous stomatitis, uveitis, genital aphthosis, pustulosis, erythema nodosum, pathergy, arthri- 
tis, thrombotic diathesis and central nervous system disease. $^{8-10}$

In a cohort of about 4000 FMF patients, we found 39 individuals from 31 families, who also suffered from BD (FMF-BD). ${ }^{11}$ With respect to their clinical and demographic characteristics, FMF-BD patients were comparable to FMF controls in the FMF arm of their disease and to BD-controls in the BD part of the disease. ${ }^{11}$ Preliminary limited mutational analysis in 27 patients with FMF-BD, looking for the most common $M E F V$ mutations ${ }^{12,13}$ by sequencing exon 10 and restriction site analysis (for the E148Q mutation), revealed that 14 patients had only one mutated MEFV (M694V mutation in most, unpublished data). This finding suggested that individuals with FMF-BD may express the FMF phenotype despite harbouring only one mutation. In the following, we test this hypothesis by sequencing the whole coding region of $M E F V$ and exploring a possible role for the non-carrier chromosome in the expression of FMF in heterozygous individuals of the FMF-BD families.

\section{Patients and methods \\ Patients}

Eight patients with FMF-BD constituted the study group. These patients came from six non-Ashkenazi Jewish families with 47 members, of whom 14 had FMF-BD, eight FMF alone, one BD alone and 24 were unaffected. The eight patients of the study group were selected based on: (1) a preliminary study, suggesting that they carry only one mutation in the coding region of MEFV (M694V in all, see Introduction); (2) the presence of other family members with FMF, BD or FMF-BD, increasing the likelihood that the families are informative; and (3) the availability of DNA from most other family members. The FMF, BD and FMFBD patients fulfilled published criteria for FMF, BD or both, respectively. ${ }^{8,11,14}$ All individuals in the current study were part of a previous study on FMF and BD, which describes in detail how the patients were recruited and clinical information obtained. ${ }^{11}$ Seven family members were deceased at the time of the present study. Their diagnosis was based on kindred report or clinic chart. None of the FMF-BD patients had other known diseases and all received medications related only to FMF-BD, including colchicine, non-steroidal anti-inflammatory drugs and low dose prednisone. Blood samples from all FMF-BD patients and 25 relatives were collected into EDTA containing blood count tubes and kept frozen $\left(-20^{\circ} \mathrm{C}\right)$ until used. Genomic DNA was extracted from the blood samples, using the High Pure PCR Template Preparation Kit (Boehringer Mannheim, Indianapolis, IN, USA). The Helsinki Committee of Sheba Medical Center approved the study.

\section{Sequence analysis}

The whole coding region of $M E F V$ of the eight patients in the study group was sequenced using primers, amplification methods, kits, automated DNA sequencer and analysis program, described elsewhere. ${ }^{1,13}$

\section{Restriction enzyme analysis}

The presence of the M694V, V726A, E148Q and P369S mutations in the $M E F V$ gene of all family members, not studied by sequence analysis, was determined using the restriction enzymes $H p h \mathrm{I}, A l u \mathrm{I}, B s t N \mathrm{I}$, and $A l u \mathrm{I}$, respectively, and the corresponding amplified DNA segment, according to previously published protocols. ${ }^{13,14}$

\section{Single nucleotide polymorphism (SNP) analysis}

The intragenic SNPs D102D (GAT $\rightarrow$ GAC) and R314R $(\mathrm{CGC} \rightarrow \mathrm{CGT})$ were studied in three families for haplotyping purposes, using the above sequence techniques. An SNP was defined by a nucleotide change, as compared to the consensus sequence of $M E F V$ and by published data, showing its common presence in unaffected control populations. ${ }^{1}$

\section{Statistical analysis}

Statistical analysis was carried out using the chi-square method.

\section{Results}

Sequence analysis of the entire $M E F V$ coding region revealed that there was indeed only one mutation (M694V) in each of the eight FMF-BD patients studied. This suggested that one chromosome, which will be designated a non-carrier chromosome, has an MEFV allele with an intact coding region. In addition, the sequence analysis revealed 10 known SNPs, present in these patients in different combinations (Table 1).

The possibility that the non-carrier chromosome causes FMF expression by a change (eg mutation) in the non-coding region of its $M E F V$ or a non-coding region related to it, was explored by looking at the association between the noncarrier chromosome and the expression of FMF in other heterozygous kin. For this aim, we studied the transmission of the carrier and non-carrier chromosomes within each family, using mutational and SNP analyses to probe the chromosomes. In three families (1, 3 and 5, Figure 1), mutational analysis revealed at least one heterozygous family member (one of the parents in each family), who shared the non-carrier chromosome with the FMF-BD proband, and yet remained unaffected.

In the other three families (2, 4 and 6, Figure 2), mutational analysis was insufficient and therefore, a combined mutational-SNP analysis was performed, using informative SNPs from those found by the sequence study of the relevant FMFBD probands (Table 1). This study showed two unaffected heterozygous sibs with the non-carrier chromosome in family 6 , and an unaffected mother in family 4 , sharing each of her two non-carrier chromosomes with a different heterozygous FMF-BD child (Figure 2). Family 2 was non- 
Table 1 Deduced MEFV-SNP haplotypes in the carrier and non-carrier chromosomes of patients with familial Mediterranean fever - Behçet's disease (FMF-BD)

\begin{tabular}{|c|c|c|c|c|c|c|c|c|c|c|c|}
\hline $\begin{array}{l}\text { Type of } \\
\text { chromosome }\end{array}$ & Patient & $\begin{array}{l}\text { Exon } 2 \\
D 102 D \\
(X 2.1) \\
G A T \rightarrow \\
G A C\end{array}$ & $\begin{array}{l}\text { G138G } \\
(X 2.2) \\
\text { GGA } \rightarrow \\
\text { GGG }\end{array}$ & $\begin{array}{l}A 165 A \\
(X 2.3) \\
G C C \rightarrow \\
G C A\end{array}$ & $\begin{array}{l}R 202 Q \\
(X 2.4) \\
C G G \rightarrow \\
C A G\end{array}$ & $\begin{array}{l}\text { Exon } 3 \\
R 314 R \\
(X 3.1) \\
\text { CGC } \rightarrow \\
\text { CGT }\end{array}$ & $\begin{array}{l}\text { Exon } 5 \\
\text { E474E } \\
(X 5.1) \\
G A G \rightarrow \\
G A A\end{array}$ & $\begin{array}{l}\text { Q476Q } \\
(X 5.2) \\
C A A \rightarrow \\
C A G\end{array}$ & $\begin{array}{l}\text { D510D } \\
(X 5.3) \\
G A T \rightarrow \\
G A C\end{array}$ & $\begin{array}{l}\text { Exon } 9 \\
\text { P588P } \\
(X 9.1) \\
C C A \rightarrow \\
C C G\end{array}$ & $\begin{array}{l}\text { Exon } 10 \\
\text { P706P } \\
\text { CCG } \rightarrow \\
C C A\end{array}$ \\
\hline \multirow[t]{2}{*}{ Carrier } & $\begin{array}{l}9.1,10.2,5.2, \\
9.4,7.5,5.6\end{array}$ & + & + & + & + & - & - & - & - & - & - \\
\hline & $4.3,5.3$ & - & - & - & - & - & - & - & - & - & + \\
\hline \multirow[t]{3}{*}{ Non-carrier } & $\begin{array}{l}10.2,9.4,7.5, \\
5.6\end{array}$ & + & + & + & + & + & + & + & + & + & - \\
\hline & $9.1,5.2$ & + & + & + & + & - & - & - & - & - & - \\
\hline & $4.3,5.3$ & - & - & - & - & - & - & - & - & - & + \\
\hline
\end{tabular}

Determination of the single nucleotide polymorphisms (SNPs) and mutations is based on the sequence of the whole coding region of the FMF gene (MEFV). All patients are carriers of the M694V mutation (exon 10). The parentheses present published SNP's nomenclature. ${ }^{1}$ Numbers of patients are according to Figures 1 and 2. The signs +/ - indicate the presence/absence of the specific SNP change. The MEFV-SNP haplotypes of the carrier chromosomes were determined based on published SNP haplotypes and their association with the M694V mutation. ${ }^{1}$ The noncarrier MEFV-SNP haplotype is deduced from the MEFV sequence analysis and the MEFV-SNP haplotypes of the homologous carrier chromosomes. Note that the non-carrier chromosomes of the eight patients with FMF-BD converge into three different haplotypes, $(+++++++++-,++++------,--------+)$, not sharing even one common SNP. This finding is not consistent with the presence of one type of mutation in the non-carrier chromosomes predisposing patients to develop the FMF-BD. Abbreviations for amino acids: A-Ala, DAsp, E-Glu, G-Gly, M-Met, P-Pro, Q-Gln, R-Arg, V-Val.

informative by the current analysis. Altogether, the mutation and SNP analyses do not support a role for the non-carrier chromosomes in FMF expression in at least six of eight heterozygous patients with FMF-BD from five of the six families studied.

Table 1 displays a deduced SNP haplotyping of the carrier and non-carrier chromosomes in the eight patients with FMF-BD. As can be seen, the eight non-carrier chromosomes converge to three MEFV-SNP haplotypes, which do not share even one common SNP per position. This finding also argues against the possibility that the expression of the FMF-BD phenotype is due to an $M E F V$ related mutation in the noncarrier chromosome, which was missed in our analysis (see Discussion).

The effect of BD on the expression of FMF phenotype in carriers of a single mutated $M E F V$ allele was determined by association analysis. In the families studied, there were 27 presumed heterozygous individuals. Of these 11 had FMF and 16 were unaffected. BD was found in 10 of the 11 heterozygous FMF patients, vs one of 16 heterozygous unaffected individuals $(P<0.001$, Table 2$)$. This finding supports a role for $\mathrm{BD}$ in the expression of the FMF phenotype in carriers of an $M E F V$ in the cohort studied.

\section{Discussion}

Sequence analysis in all eight FMF-BD patients from the six families studied confirmed that there is only one FMF associated mutation (M694V) in the MEFV coding region of each patient. The possibility that the non-carrier chromosome of these patients precipitated FMF perhaps by changes in intra- or extra-genic non-coding regions, is unlikely in at least six patients sharing the non-carrier chromosome with heterozygous kin, who nevertheless remain clinically unaffected (Figures 1 and 2). Family 2 with two FMF-BD patients, in whom the role of the non-carrier chromosome could not be determined by the analysis employed, should be regarded as a non-informative family. These results suggest that despite the known recessive mode of inheritance, FMF may be expressed in individuals carrying only one mutated $M E F V$ allele. Alternatively, these findings may be explained by the presence of a low penetrance mutation, not detected by us, in non-coding regions (eg introns, promoter, enhancer etc.), which causes FMF expression only in a fraction of its carriers.

The finding that the non-carrier chromosomes of the eight FMF-BD patients converged into three different $M E F V$ haplotypes, not sharing even one SNP site (Table 1), argues against the possibility of a 'missed' low penetrance mutation. This notion is based on the findings that within the gene and its close vicinity, the number of haplotypes becomes limited and ethnic related. ${ }^{1,13}$ One therefore may assume that in a restricted ethnic population, characterised by a specific (FMF$\mathrm{BD})$ phenotype, there would be only one $M E F V$ haplotype carrying only one 'missed' mutation, or that all different haplotypes will converge to the same SNP typing in at least one region of the gene.

The finding that all carriers of a mutated FMF gene who express $\mathrm{FMF}$, except one, also have $\mathrm{BD}$, while all carriers, except one, who do not have $\mathrm{BD}$, remain unaffected (Table 2), suggests that BD may induce the expression of FMF in carriers. Alternatively, if the underlying mechanism is an unidentified low-penetrance mutation, the latter finding suggests that BD may lead to the expression of this mutation. The two $M E F V$ carriers expressing pure FMF or pure BD (Patients 3.3 and 2.4 respectively, Figures 1 and 2), who do 

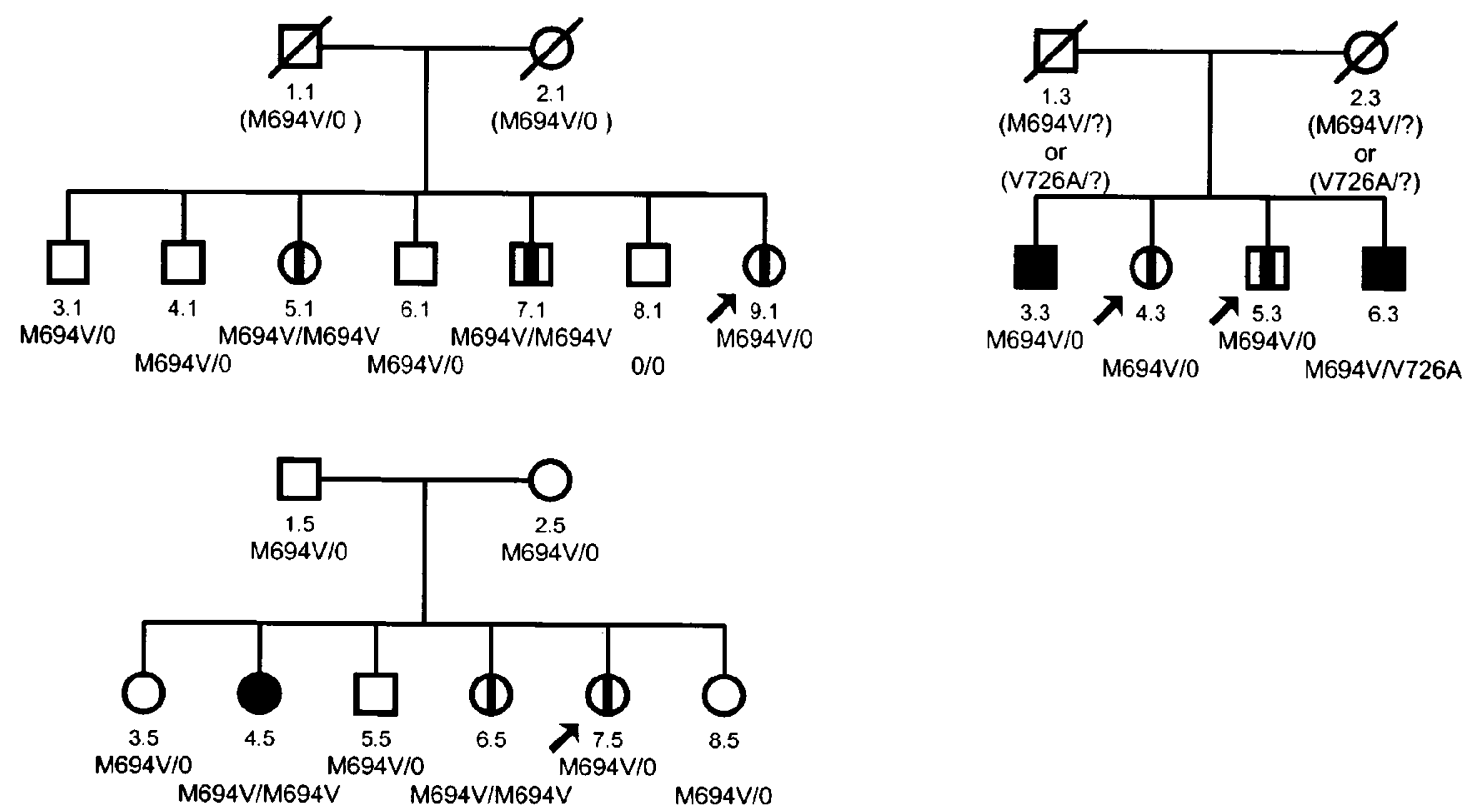

Figure 1 Mutational analysis in three families with familial Mediterranean fever - Behçet's disease (FMF-BD). The analysis is based on sequence determination in the patients marked by arrows and on the study of four mutations, M694V, V726A, E148Q and P369Q, using endonuclease restriction site determination, in other family members. Open/closed boxes and circles indicate unaffected/FMF individuals respectively. Vertical line in the centre of an open box or a circle indicates a patient with FMF-BD. Crossed box/circle denotes dead kin. The unstudied, putative (deduced) mutations are depicted in parentheses. Question mark implies that the mutation is not deducible. Zero mutation means that no second mutation was found. The first of the two digits identifying the patients is the number of the patient within the family and the second is the number of the family. In all families shown, the parents are unaffected and have at least one mutation in their MEFV. In each family, one of the parents must be the origin of the non-carrier chromosome. Therefore, the absence of FMF in any parent supports the possibility that the expression of FMF in the proband with FMF-BD is not associated with an abnormality in the non-carrier chromosome.

not conform to the hypothesis, probably express phenotypes determined by other enhancing or restraining factors.

The pathogenesis of FMF expression in this setting however is yet to be determined. The elucidation of a possible role for BD-related genetic factors is precluded, partly by the absence of known BD genes. In addition, previous analysis, showing that only about $50 \%$ of all our FMF-BD patients and a comparable rate of BD controls carried one of the HLA-B5 antigens, is not in favour of such an association. ${ }^{11}$ MICA however, another BD associated HLA class I antigen, ${ }^{15}$ was recently identified as a possible modifier gene, influencing the severity of FMF in certain patients. ${ }^{16}$ Another possible underlying mechanism may be traced to granulocytes, the effector cells in both conditions and the cells in which pyrin is almost exclusively expressed. ${ }^{1}$ Thus, one may hypothesise that a reduced level of functional pyrin, presumably characterising heterozygosity, on the one hand, and increased demand for pyrin, by activated granulocytes of $\mathrm{BD}$, on the other, may ultimately lead to FMF attacks.

BD may be only one example of various inflammatory, infectious and other stimuli, possibly predisposing carriers of a mutated FMF gene to acute attacks of FMF. Bouts of FMF in association with active tuberculosis were reported in a patient carrying only one FMF mutation. ${ }^{17}$ In another report, unaffected first-degree relatives of FMF patients, mostly obligatory carriers of a mutated FMF gene, showed significantly elevated serum levels of acute phase reactants. ${ }^{18} \mathrm{~A}$ third study in two Turkish families, showing that FMF may not map to chromosome $16,{ }^{19}$ may also be consistent with the expression of FMF in carriers. A recent contribution by Booth et al displayed dominant inheritance (FMF in carriers) in three families with FMF who harboured a deletion in M694 or had a M694I-E148Q complex allele. ${ }^{20}$ Most impressive is the finding of an increasing number of FMF patients, in whom only one mutation has been identified, despite sustaining a typical clinical picture..$^{5,12,13,21-24}$ While most of these instances are hampered by a limited genetic analysis, or by an incomplete clinical evaluation, at least in three, a complete sequence of the coding region was performed. ${ }^{5,23,24}$ Our findings not only agree with these studies, but also suggest that FMF expression most likely occurs in the absence of defects in the non-carrier chromosome.

Of all SNPs found in the MEFV of patients with FMF-BD, the R202Q SNP is the only one causing amino acid substitution (Table 1). Therefore, its effect on the expression of FMF is of interest. The substitution caused by this SNP was considered 

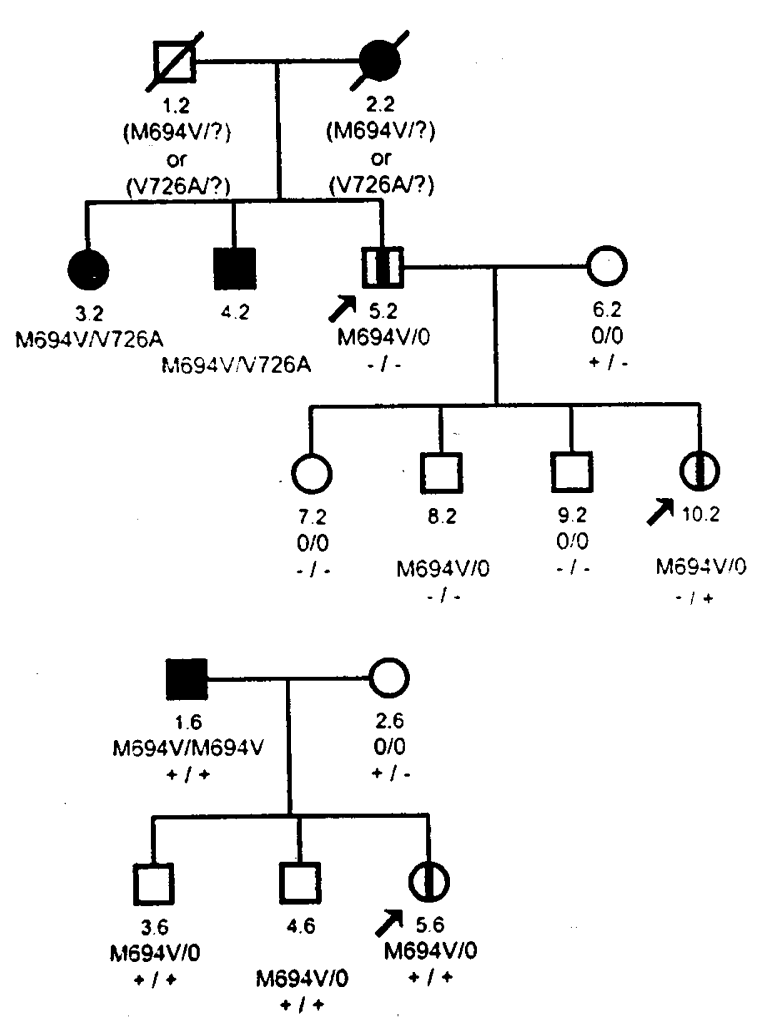

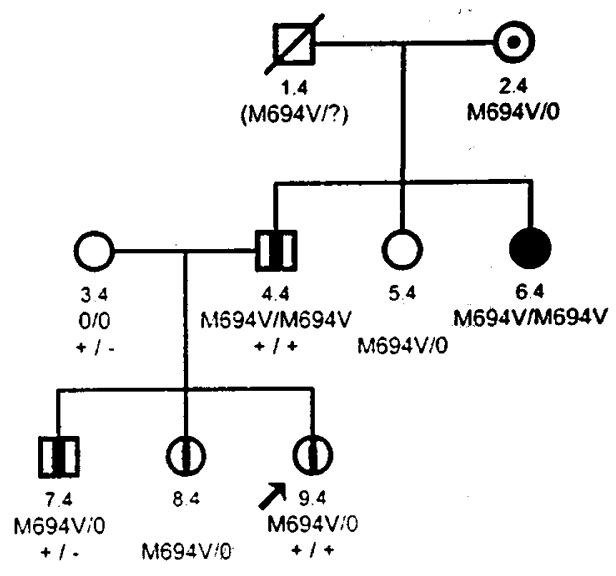

Figure 2 Combined mutation-single nucleotide polymorphism (SNP) analysis in three families with familial Mediterranean fever Behçet's disease (FMF-BD). The mutational analysis is similar to that described in Figure 1 . The SNPs were determined by sequencing exon 3 and 2, looking for the SNP R314R (CGC $\rightarrow$ CGT) in family 2 (family members 6.2, 7.2, 8.2, 9.2) and the SNP D102D (GAT $\rightarrow$ GAC) in families 4 and $6(3.4,4.4,7.4,1.6,2.6,3.6,4.6)$. The signs $+/$ - below the mutation, indicate the presence/absence of the relevant SNP in the homologous chromosomes of the patient. A patient with BD alone (2.4) is marked by a dot in the centre of the open circle. All other signs are as in Figure 1. The analysis reveals in family 6 two heterozygous sibs, 3.6 and 4.6, who share with the proband 5.6 the non-carrier chromosome, and in family 4 a mother (3.4), who shares a different non-carrier chromosome with each of her two FMF affected offspring 9.4 and 7.4. Neither the sibs, nor the mother had FMF, declining an association between the non-carrier chromosome and the expression of FMF in the probands of these families. Family 2 is the only one showing phenotype-genotype concordance, and therefore the possibility that FMF expression in the probands 5.2 and 10.2 of this family is related to the non-carrier chromosome cannot be excluded by the current analysis.

Table 2 FMF in carriers is correlated with the cooccurrence of $\mathrm{BD}$

\begin{tabular}{lccc}
\hline Carrier's phenotype & $B D+$ & $B D-$ & Total \\
\hline FMF+ & 10 & 1 & 11 \\
FMF- & 1 & 15 & 16 \\
Total & 11 & 16 & 27 \\
\hline
\end{tabular}

Data is based on Figures 1 and 2. As can be inferred, the six studied families have 27 kin who are carriers of MEFV mutation. Of these, 11 have FMF while 16 are unaffected. Ten of the 11 FMF patients compared with one of the 16 unaffected individuals also have BD $(P<0.001)$. For abbreviations see Table 1 .

by the International Consortium of FMF and by Bernot et al to be phenotypically insignificant, since in both studies it was found to be highly prevalent among normal and noncarrier chromosomes, with a frequency comparable to that found in carrier chromosomes. ${ }^{1,25}$ The frequency of the non- carrier chromosomes with the R202Q SNP in heterozygous FMF patients in the families studied (eight of 11) is comparable to that found in the non-carrier chromosomes by the international consortium $(10 / 24, P<0.1)$, whose patients' origin was similar to that of our patients. Moreover, the R202Q SNP was present in 13 of 16 non-carrier chromosomes of heterozygous unaffected individuals $(P<0.7$, FMF $v s$ unaffected, data not shown). These suggest that the R202Q SNP in the non-carrier chromosomes does not predispose carriers of mutated $M E F V$ to the development of FMF.

In conclusion, our findings suggest that at least in some patients suffering from FMF-BD, the FMF is expressed despite the presence of only one coding $M E F V$ mutation and an uninvolved non-carrier chromosome. This finding was highly associated with a simultaneous occurrence of BD in these patients. Based on preliminary published reports and findings of other FMF patients with only one known 
mutation, it is possible that our finding in FMF-BD reflects a more general rule that not all patients with FMF conform to a recessive mode of inheritance.

\section{References}

1 The International FMF Consortium: Ancient missense mutations in a new member of the RoRet gene family are likely to cause familial Mediterranean fever. Cell 1997; 90: 797-807.

2 The French FMF Consortium: A candidate gene for familial Mediterranean fever. Nature Genet 1997; 17: 25-31.

3 Sohar E, Gafni J, Pras M, Heller H: Familial Mediterranean fever. A survey of 470 cases and review of the literature. Am J Med 1967; 43: $227-253$.

4 Livneh A, Langevitz P, Zemer D et al: The changing face of familial Mediterranean fever. Semin Arthritis Rheum 1996; 26: $612-627$.

5 Samuels J, Aksentijevich I, Torosyan Y et al: Familial Mediterranean fever at the millennium. Clinical spectrum, ancient mutations and a survey of 100 American referrals to the National Institute of Health. Medicine 1998; 77: 268-297.

6 O’Duffy JD: Behçet's disease. Curr Opin Rheumatol 1994; 6: 39 43.

7 Brautbar C, Chajek T, Ben-Tuvia S, Lamm L, Cohen T: A genetic study of Behçet disease in Israel. Tissue Antigens 1978; 11: 113 120.

8 International Study Group for Behçet's disease: Criteria for diagnosis of Behçet's disease. Lancet 1990; 335: 1078-1080.

9 Shimizu T, Ehrlich GE, Inaba G, Hayashi K: Behçet disease (Behçet syndrome). Semin Arthritis Rheum 1979; 8: 223-260.

10 Chajek T, Fainaru M: Behçet's disease. Report of 41 cases and a review of the literature. Medicine 1975; 54: 179-196.

11 Schwartz T, Langevitz P, Zemer D, Gazit E, Pras M, Livneh A: Behçet's disease in familial Mediterranean fever: characterization of the association between the two diseases. Semin Arthritis Rheum 2000; 29: 286-295.

12 Cazeneuve C, Sarkisian T, Pêcheux C et al: MEFV-gene analysis in Armenian patients with familial Mediterranean fever: Diagnostic value and unfavorable renal prognosis of the M694V homozygous genotype - genetic and therapeutic implications. Am J Hum Genet 1999; 65: 88-97.

13 Aksentijevich I, Torosyan Y, Samuels J et al: Mutation and haplotype studies of familial Mediterranean fever reveal new ancestral relationships and evidence for high carrier frequency with reduced penetrance in the Ashkenazi Jewish population. Am J Hum Genet 1999; 64: 949-962.
14 Livneh A, Langevitz P, Zemer D et al: Criteria for the diagnosis of familial Mediterranean fever. Arthritis Rheum 1997; 40: 18841890.

15 Ota M, Mizuki N, Katsuyama Y et al: The critical region for Behçet's disease in the human major histocompatibility complex is reduced to a $46-\mathrm{kb}$ segment centromeric of HLA-B, by association analysis using refind microsatellite mapping. Am J Hum Genet 1999; 64: 1406-1410.

16 Touitou I: The spectrum of FMF mutations. Second International Conference on FMF. Book of Lectures and Abstracts 2000: $23-26$.

17 Holmes AH, Booth DR, Hawkins PN: Familial Mediterranean fever gene. N Engl J Med 1998; 338: 992-993.

18 Tunca M, Kirkali G, Soytürk M, Akar S, Pepys MB, Hawkins PN: Acute phase response and evolution of familial Mediterranean fever. Lancet 1999; 353: 1415.

19 Akarsu AN, Saatci U, Ozen S, Bakkaloglu A, Besbas N, Sarfarazi M: Genetic linkage study of familial Mediterranean fever (FMF) to $16 \mathrm{p} 13.3$ and evidence for genetic heterogeneity in the Turkish population. J Med Genet 1997; 34: 573-578.

20 Booth DR, Gillmore JD, Lachmann HJ et al: The genetic basis of autosomal dominant familial Mediterranean fever. Q $J$ Med 2000; 93: $217-221$

21 Tamir N, Langevitz P, Zemer D et al: Late onset familial Mediterranean fever (FMF): A subset with distinct clinical, demographic, and molecular genetic characteristics. Am J Med Genet 1999; 87: 30-35.

22 Eisenberg S, Aksentijevitch I, Deng Z, Kastner DL, Matzner Y: Diagnosis of familial Mediterranean fever by a molecular genetics method. Ann Intern Med 1998; 129: 539-542.

23 Press J, Shinar Y, Langevitz P, Livneh A, Pras M, Buskila D: Familial Mediterranean fever in two Bedouin families: Mutation analysis and disease severity. Am J Med Genet 2000; 92: 247 - 249.

24 Domingo C, Touitou I, Bayou A et al: Familial Mediterranean fever in the 'Chuetas' of Mallorca: a question of Jewish origin or genetic heterogeneity. Eur J Hum Genet 2000; 8: 242-246.

25 Bernot A, da Silva C, Petit J-L et al: Non-founder mutations in the MEFV gene establish this gene as the cause of familial Mediterranean fever (FMF). Hum Mol Genet 1998; 7: 1317 - 1325. 